\title{
PHYSIK AM ENEM UND IM KURS FÜR TECHNISCHE CHEMIE AN DER BUNDESANSTALT AMAPÁ (IFAP): EIN LEHRPLANVERGLEICH
}

\section{ORIGINALER ARTIKEL}

CARMO, Denny Rodrigues do ${ }^{1}$, BASTOS, Argemiro Midonês², FECURY, Amanda Alves $^{3}$, DENDASCK, Carla Viana ${ }^{4}$, OLIVEIRA, Euzébio de ${ }^{5}$, DIAS, Claudio Alberto Gellis de Mattos ${ }^{6}$

CARMO, Denny Rodrigues do. Et al. Physik am ENEM und im Kurs für Technische Chemie an der Bundesanstalt Amapá (IFAP): Ein Lehrplanvergleich. Revista Científica Multidisciplinar Núcleo do Conhecimento. Jahr 06, Ed. 03, Vol. 03, pp. 80-88. März 2021. ISSN: 2448-0959, Zugangslink: https://www.nucleodoconhecimento.com.br/bildung-de/physik-am-enem, DOI: 10.32749/nucleodoconhecimento.com.br/bildung-de/physik-am-enem

\section{ABSTRAKT}

Die National High School Examination (ENEM) ist ein bewertendes und selektives Instrument für Schüler, um in die Hochschulbildung einzutreten. Die Bundesinstitute für Bildung, Wissenschaft und Technologie (Fls) sind von der Bundesregierung

\footnotetext{
${ }^{1}$ Student des Technischen Kurses in Chemie (Gymnasium) am Institut für grundlegende, technische und technologische Ausbildung von Amapá (IFAP).

${ }^{2}$ Physiker, PhD in Biodiversität und Biotechnologie (UFPA), Professor und Forscher des Physik-Studiengangs am Basic, Technical and Technological Institute von Amapá (IFAP) und des Graduiertenprogramms für berufliche und technologische Ausbildung (PROFEPT IFAP).

${ }^{3}$ Biomedizin, PhD in topischen Krankheiten, Professor und Forscher des Medizinkurses am Campus Macapá der Bundesuniversität Amapá (UNIFAP).

${ }^{4}$ Theologe, PhD in klinischer Psychoanalyse. Er hat 15 Jahre mit Scientific Methodology (Research Method) in der Orientierung der wissenschaftlichen Produktion von Master- und Doktoranden gearbeitet. Spezialist für Marktforschung und Forschung mit Schwerpunkt Gesundheit.

${ }^{5}$ Biologe, PhD in Topical Diseases, Professor und Forscher des Sportlehrgangs an der Federal University of Pará (UFPA).

${ }^{6}$ Biologe, Doktor in Verhaltenstheorie und -forschung, Professor und Forscher des Chemie-Studiengangs am Institut für grundlegende, technische und technologische Ausbildung von Amapá (IFAP) und des Graduiertenkollegs für berufliche und technologische Ausbildung (PROFEPT IFAP).
}

RC: 78530

Disponível em: https://www.nucleodoconhecimento.com.br/bildung-de/physik-am- 
geschaffene Einrichtungen mit dem Ziel, kompetente Fachkräfte auszubilden. Ziel dieser Studie ist es, den Inhalt der Physikfragen der National High School Examination (ENEM) zwischen 2014 und 2018 mit dem Lehrplaninhalt des Kurses Technische Chemie an der Bundesanstalt Amapá (IFAP) zu vergleichen. Der Physikunterricht im technischen Studiengang Chemie am IFAP stellt keine Abteilung dar, die die aktuellsten Fächer in ENEM priorisiert. ENEM kontextualisiert normalerweise seine Fragen. Dies könnte eine gängige Praxis in der Physik der High School sein, da dies zu Ihrem besseren Verständnis beitragen würde. Darüber hinaus ist es notwendig, den Inhalt weder während des Unterrichts noch als Komposition mit anderen Fächern zu fragmentieren. Der technische Kurs am IFAP wäre nicht der geeignete Studienort für diejenigen, die nur die High School beenden möchten. Der Inhalt geht über das Erforderliche hinaus, jedoch mit Ausschnitten, die auf den technischen Teil ausgerichtet sind, einschließlich Laborpraktiken und streng technischer Disziplinen. Das Fehlen von Interdisziplinarität und Kontextualisierung macht es schwierig, den Inhalt aufzunehmen, was zu Studenten führt, die Schwierigkeiten haben, über Physik nachzudenken.

Schlüsselwörter: ENEM, EPT, Lehre, Physik, IFAP.

\section{EINFÜHRUNG}

Die National High School Exam (ENEM) wurde 1998 von der brasilianischen Bundesregierung ins Leben gerufen und zielte darauf ab, Schüler zu bewerten, die die High School abgeschlossen haben. Im Laufe der Zeit gewann ENEM an Relevanz, als es nicht nur als Bewertungsinstrument, sondern auch als selektives Instrument für den Einstieg von Studierenden in die Hochschulbildung eingesetzt wurde (Silveira et al., 2015).

Im ENEM-Test wird das Fach Physik zusammen mit den Fächern Biologie und Chemie im Bereich der Wissenswissenschaft der Natur behandelt. Obwohl es keine formale Trennung gibt, ist es möglich zu sehen, dass die Fragen der

RC: 78530

Disponível em: https://www.nucleodoconhecimento.com.br/bildung-de/physik-am- 
Naturwissenschaften durch Analyse der behandelten Inhalte zwischen den drei Disziplinen organisiert werden können. (Silveira , 2015).

Die Bundesinstitute für Bildung, Wissenschaft und Technologie sind von der Bundesregierung geschaffene Einrichtungen mit dem Ziel, kompetente Fachkräfte auszubilden. Diese können verschiedene Kurse wie Bachelor-Abschlüsse, MasterAbschlüsse oder Promotionen beinhalten, aber die Hauptmodalität ist die technische Ausbildung, hauptsächlich die technische Ausbildung, die in die High School integriert ist (Pacheco, 2010).

In Brasilien haben alle Bundesländer mindestens ein Bundesinstitut, einige sogar mehr als ein Bundesinstitut mit insgesamt 38 Einheiten. Ein Bundesinstitut kann in Standorte unterteilt werden, von denen derzeit insgesamt 644 in Betrieb sind. (Brasil, 2018).

Die Bundesanstalt für Bildung, Wissenschaft und Technologie des Staates Amapá (IFAP) wurde am 28 Dezember 2008 mit dem Gesetz Nr. 11.892 (Marques et al., 2020) gegründet und soll zur Entwicklung des Staates beitragen (Castro et al., 2020). IFAP bietet Kurse an, die von der Hochschulbildung bis zur High School reichen, und schafft es damit, ein breites Publikum auf verschiedenen Ebenen zu erreichen. Es besteht auch aus mehreren Campus, von denen wir den Macapá-Campus in der Hauptstadt erwähnen können (Brasil, 2019) ; Penha et al., 2020).

Der Macapá-Campus bietet den chemisch-technischen Kurs der High School in integrierter Form an. In diesem Kurs haben wir die Lehrplankomponenten der National Common Base, die sich auf die Bereiche Sprachen, Mathematik, Geistesund Naturwissenschaften beziehen. Im Bereich der Naturwissenschaften haben wir die Lehrplankomponente der Physik, die während der drei Jahre des Kurses behandelt wird. Jedes Jahr hat eine Arbeitsbelastung von 80 Stunden pro Jahr und die unterrichteten Inhalte sind in vier Einheiten unterteilt, insgesamt 12 Einheiten in den drei Jahren. (Brasil, 2016).

$\mathrm{RC}: 78530$

Disponível em: https://www.nucleodoconhecimento.com.br/bildung-de/physik-am- 
Am Bundesinstitut Amapá (IFAP) sind die Themen des Menüs der Komponente "Physik“ des ersten Jahres des integrierten integrierten technischen Kurses: Einführung in Physik und Kinematik; Dynamik; Arbeit und Energie (einschließlich Bewegung und Impuls); Gravitations- und Flüssigkeitsstatik. Im zweiten Jahr umfasst der Lehrplan: Wärme und Temperatur; Thermodynamik; Geometrische Optik; Oszillationen und Wellen (einschließlich einfacher harmonischer Bewegung). Im dritten und letzten Jahr sind die Inhalte: Elektrostatisch; Elektrodynamik; Magnetismus; und Elektromagnetismus (Brasil, 2016).

Innerhalb der Enem-Lehrplanmatrix gibt es Kompetenzen und Wissensobjekte, die mit diesen Kompetenzen zusammenhängen. Wissensobjekte in Bezug auf Physik werden in 7 Themen gesammelt: Grund- und Grundwissen (einschließlich Fächer der Dimensionsanalyse und Einheitensysteme); Bewegung, Gleichgewicht und die Entdeckung physikalischer Gesetze (einschließlich Kinematik, Dynamik, Hydrostatik, Statik, Impuls und Bewegungsmenge); Energie, Arbeit und Energie (einschließlich Angelegenheiten im Zusammenhang mit Arbeit und Energie); die Mechanik und Funktionsweise des Universums (einschließlich Themen im Zusammenhang mit Gravitation); elektrische und magnetische Phänomene (mit Themen im Zusammenhang mit Elektrizität und Magnetismus); Schwingungen, Wellen, Optik und Strahlung (einschließlich Motive von Optik bis Welle); sowie Wärme- und Wärmephänomene (einschließlich thermologischer Themen) (Brasil, 2015).

\section{TORE}

Vergleichen Sie den Inhalt der Physikfragen der National High School Exam (ENEM) zwischen 2014 und 2018 mit dem Lehrplaninhalt des Kurses Technische Chemie an der Bundesanstalt Amapá (IFAP).

RC: 78530

Disponível em: https://www.nucleodoconhecimento.com.br/bildung-de/physik-am- 


\section{MATERIAL UND METHODEN}

Die Forschung wurde unter Verwendung von Fragen aus der National High School Examination (ENEM) durchgeführt, die dem Superprofessor des Programms (Software) entnommen wurden, der von der Firma Interbits Computing (https://www.sprweb.com.br/mod_superpro/index.php) erworben wurde ). Die körperliche Disziplin und die Jahre 2014 bis 2018 wurden im Programm ausgewählt. Die Fragen wurden nach dem Programm klassifiziert (nach Analyse und Entfernung überlappender Fragen). Anschließend wurde ein Vergleich zwischen diesen und dem Lehrplan der physikalischen Komponente des technischen Kurses der Bundesanstalt Amapá (IFAP) durchgeführt. Die bibliografische Recherche wurde an wissenschaftlichen Artikeln auf Computern des Instituts des Bundesinstituts für Bildung, Wissenschaft und Technologie von Amapá, Campus Macapá, Rodovia BR 210 KM 3, s / n - Bairro Brasil Novo, durchgeführt. CEP: 68.909-398, Macapá, Amapá, Brasilien. Die Daten wurden in der Excel-Anwendung kompiliert, die Teil der Office-Suite der Microsoft Corporation ist.

\section{ERGEBNISSE}

Die Klassifizierung des überlappenden Inhalts von ENEM-Physikfragen zwischen 2014 und 2018 nach Menge und Prozentsatz der Fragen ist in Tabelle 1 aufgeführt. Drei (3) Probanden stellen keine Frage (Dimensionsanalyse / Einheitensystem, Gravitation und moderne Physik). Die meisten Fächer haben eine (1) oder zwei (2) Fragen. Elektrizität + Magnetismus und Kinematik stellen jeweils 4 Fragen. Sechs (6) der Fragen beziehen sich auf das Thema Arbeit und Energie sowie auf das Thema Optik. Das Thema Wave umfasst insgesamt 17 Fragen.

$\mathrm{RC}: 78530$

Disponível em: https://www.nucleodoconhecimento.com.br/bildung-de/physik-am- 
Tabelle 1 Klassifizierung des überlappenden Inhalts von ENEM-Physikfragen zwischen 2014 und 2018 nach Menge und Prozentsatz der Fragen.

\begin{tabular}{|c|c|c|}
\hline \multicolumn{3}{|c|}{ Classificação do autor após análise de conteúdo sobreposto } \\
\hline Conteúdo de Física & Quantidade & Porcentagem \\
\hline Análise Dimensional / Sistemas de Unidades & 0 & $0 \%$ \\
\hline Gravitação & 0 & $0 \%$ \\
\hline Moderna & 0 & $0 \%$ \\
\hline Cinemática + Impulso e Quantidade de Movimento & 1 & $2 \%$ \\
\hline Dinâmica + Impulso e Quantidade de Movimento & 1 & $2 \%$ \\
\hline Hidrodinâmica & 1 & $2 \%$ \\
\hline Hidrostática & 1 & $2 \%$ \\
\hline Trabalho e Energia + Movimento Harmônico & 1 & $2 \%$ \\
\hline Temática + Trabalho e energia & 1 & $2 \%$ \\
\hline Tematica & 1 & $2 \%$ \\
\hline Cinemática + Dinâmica & 2 & $3 \%$ \\
\hline Dinâmica & 2 & $3 \%$ \\
\hline Estática & 2 & $3 \%$ \\
\hline Impulso e Quantidade de Movimento & 2 & $3 \%$ \\
\hline Trabalho e Energia + termologia & 2 & $3 \%$ \\
\hline Eletricidade + Magnetismo & 4 & $7 \%$ \\
\hline Cinemática & 4 & $7 \%$ \\
\hline Trabalho e Energia & 6 & $10 \%$ \\
\hline Óptica & 6 & $10 \%$ \\
\hline Termologia & 7 & $11 \%$ \\
\hline Ondulatória & 17 & $28 \%$ \\
\hline TOTAIS & 61 & $100 \%$ \\
\hline
\end{tabular}

Tabelle 2 zeigt die Themen des Physikmenüs des technischen Kurses in Chemie am IFAP mit der Einheitennummer (pro Bimonth) und dem Jahr, das abgedeckt wird. Die Einheiten I bis IV müssen in achtzig (80) Unterrichtsstunden unterrichtet werden. Im ersten Jahr werden normalerweise 12 Stunden verwendet, um Einheit I zu unterrichten, 28 Stunden, um Einheit II zu unterrichten, 20 Stunden für Einheit III und 20 Stunden für Einheit IV. Im zweiten Jahr wird Einheit I in der Regel in 20 Stunden

$\mathrm{RC}: 78530$

Disponível em: https://www.nucleodoconhecimento.com.br/bildung-de/physik-amenem 
unterrichtet, ebenso wie jede der späteren Einheiten (II, III und IV). Im dritten Jahr ist Einheit I in 16 Stunden abgeschlossen, Einheit II in 36 Stunden, Einheit III in 14 Stunden und Einheit IV in 14 Stunden.

Tabelle 2. Themen des Physikmenüs des technischen Kurses in Chemie am IFAP nach Jahr und Einheit.

\begin{tabular}{|c|c|c|c|c|c|c|}
\hline \multirow[b]{2}{*}{ Unidade } & \multicolumn{6}{|c|}{ Ano } \\
\hline & $1^{\circ}$ Ano & $\begin{array}{c}\mathrm{n}^{\circ} \text { de horas } \\
\text { aulas }\end{array}$ & $2^{\circ}$ Ano & $\begin{array}{c}\mathrm{n}^{\circ} \text { de horas } \\
\text { aulas }\end{array}$ & $3^{\circ}$ Ano & $\begin{array}{c}\mathrm{n}^{\circ} \text { de horas } \\
\text { aulas }\end{array}$ \\
\hline I & $\begin{array}{l}\text { Introdução à } \\
\text { Fisica e } \\
\text { Cinemática } \\
\end{array}$ & 12 & $\begin{array}{c}\text { Calore } \\
\text { Temperatura }\end{array}$ & 20 & Eletrostática & 16 \\
\hline II & \begin{tabular}{|c|} 
Dinâmica \\
(evidenciando o \\
conceito de força \\
e aplicações) \\
\end{tabular} & 28 & Termodinâmica & 20 & Eletrodinâmica & 36 \\
\hline III & $\begin{array}{c}\text { Trabalho e } \\
\text { Energia }\end{array}$ & 20 & $\begin{array}{c}\text { Óptica } \\
\text { Geométrica }\end{array}$ & 20 & Magnetismo & 14 \\
\hline IV & $\begin{array}{c}\text { Gravitação e } \\
\text { Estática dos } \\
\text { Fluidos }\end{array}$ & 20 & $\begin{array}{c}\text { Oscilações e } \\
\text { Ondas }\end{array}$ & 20 & Eletromgnetismo & 14 \\
\hline $\begin{array}{l}\mathrm{n}=\text { aulas da } \\
\text { matriz anual }\end{array}$ & & 80 & & 80 & & 80 \\
\hline
\end{tabular}

Die vereinfachte Klassifizierung des Inhalts von ENEM-Physikfragen zwischen 2014 und 2018 nach Menge und Prozentsatz der Fragen ist in Tabelle 3 dargestellt. Das thematische Thema erscheint in $2 \%$ der Fragen, gefolgt vom Thema Elektrizität + Magnestimo (7\%). Optik (10\%), Thermologie (11\%); Welle (28\%) und Mechanik $(43 \%)$.

$\mathrm{RC}: 78530$

Disponível em: https://www.nucleodoconhecimento.com.br/bildung-de/physik-am- 
Tabelle 3. Vereinfachte Klassifizierung des Inhalts von ENEM-Physikfragen zwischen 2014 und 2018 nach Menge und Prozentsatz der Fragen.

\begin{tabular}{l|c|c|}
\hline Assunto & Quantidade & Porcentagem \\
\hline Tematica & 1 & $2 \%$ \\
\hline Eletricidade + Magnetismo & 4 & $7 \%$ \\
\hline Óptica & 6 & $10 \%$ \\
\hline Termologia & 7 & $11 \%$ \\
\hline Ondulatória & 17 & $28 \%$ \\
\hline Mecânica & 26 & $43 \%$ \\
\hline Total & $\mathbf{6 1}$ & $\mathbf{1 0 0} \%$ \\
\hline
\end{tabular}

Die vereinfachte Klassifizierung des Inhalts von ENEM-Physikfragen zwischen 2014 und 2018, geteilt durch das Jahr, in dem sie im technischen Kurs für Chemie am IFAP unterrichtet werden, ist in Tabelle 4 aufgeführt. Der angezeigte Prozentsatz bezieht sich auf den Teil der ENEM-Fragen innerhalb jedes Thema.

Tabelle 4. Vereinfachte Klassifizierung des Inhalts von ENEM-Physikfragen zwischen 2014 und 2018, geteilt durch das Jahr, in dem sie im technischen Kurs für Chemie am IFAP unterrichtet werden

\begin{tabular}{|c|c|c|c|c|}
\hline & \multicolumn{4}{|c|}{ Curso técnico em química do IFAP } \\
\hline & $1^{\circ}$ & $2^{\circ}$ & $3^{a}$ & Todos \\
\hline \multirow{3}{*}{$\begin{array}{l}\text { Assuntos com } \\
\text { as porcentagem } \\
\text { de questões }\end{array}$} & \multirow{3}{*}{ Mecânica (43\%) } & Óptica $(10 \%)$ & \multirow{3}{*}{$\begin{array}{c}\text { Eletricidade }+ \\
\text { Magnestismo (7\%) }\end{array}$} & \multirow{3}{*}{ Tematica $(2 \%)$} \\
\hline & & Termologia (11\%) & & \\
\hline & & Ondulatória (28\%) & & \\
\hline $\begin{array}{c}\text { Porcentagem } \\
\text { Total }\end{array}$ & $43 \%$ & $49 \%$ & $7 \%$ & $100 \%$ \\
\hline
\end{tabular}

RC: 78530

Disponível em: https://www.nucleodoconhecimento.com.br/bildung-de/physik-am- 
Abbildung 1 zeigt die Anzahl der Physikfragen bei ENEM zwischen 2014 und 2018 nach Schwierigkeitsgrad. Die vom Superprofessor-Programm als niedrig eingestuften Fragen nahmen von 2014 bis 2016 mengenmäßig ab und nahmen von 2016 bis 2018 wieder zu. Die Fragen mit mittlerem Schwierigkeitsgrad nahmen von 2014 bis 2016 zu und von 2016 bis 2018 ab. Die als hoch eingestuften Fragen Schwierigkeit erscheinen nur im Jahr 2015.

Abbildung 1 - Anzahl der Physikfragen bei ENEM zwischen 2014 und 2018 nach Schwierigkeitsgrad

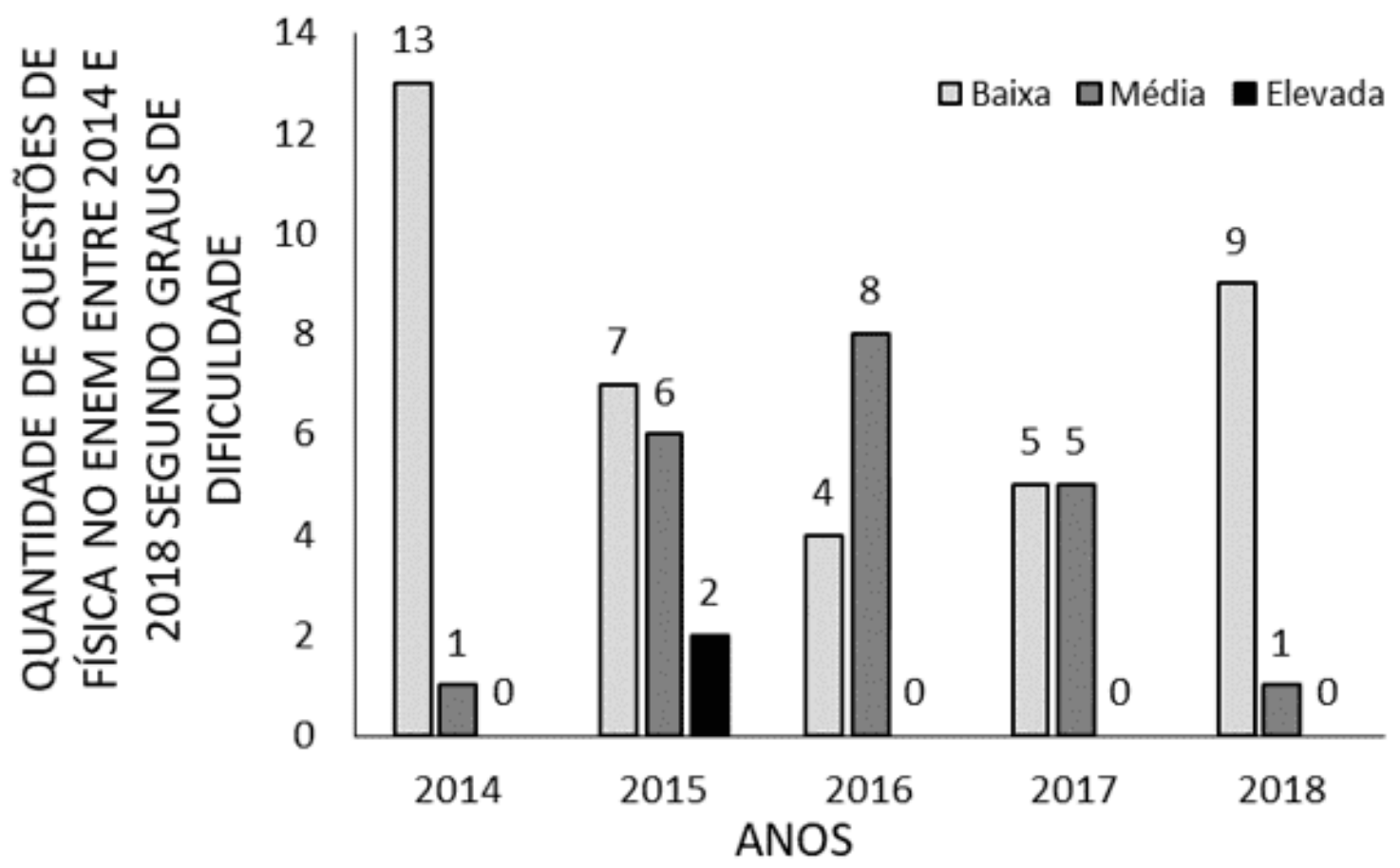

\section{DISKUSSION}

Die National High School Exam hat als Parameter einen Kontext, um die Fragen der körperlichen Untersuchung zu kontextualisieren, die sie mit dem täglichen Leben der Menschen verbinden (Silveira et al., 2014). In diesem Sinne kann der Unterschied zwischen den Probanden der Physikbewertung im untersuchten Zeitraum (Tabelle 1)

RC: 78530

Disponível em: https://www.nucleodoconhecimento.com.br/bildung-de/physik-am- 
als Folge dieser Kontextualisierung aufgetreten sein. In einer Studie über das Vorhandensein von Physik im täglichen Leben von Studenten wurden Fragen zum Einsatz verschiedener Energien (elektrisch, chemisch) in Situationen wie Alkoholproduktionsanlagen und Orten für die Installation von Tonanlagen in Kraftfahrzeugen (Toti und Pierson, 2010). Optik taucht auch routinemäßig im Leben der Menschen auf. Das Studium des Lichts kann einem Ingenieur helfen, zu wissen, wie viel Schatten sein entworfenes Gebäude in der Nachbarschaft verursachen wird. oder wie eine Tasse oder ein metallischer Gegenstand lesen Sie ein Bild; oder sogar, weil der Himmel blau ist, wenn der Tag sonnig ist (Ribeiro und Verdeaux, 2012).

Im technischen Kurs wird im Rahmen des Elektromagnetismus eine Einführung in die spezielle Relativitätstheorie gegeben, die Gegenstand der modernen Physik ist (Brasil, 2016). Die moderne Physik in der High School ist noch kein konsolidierter Inhalt und es besteht kein Konsens über ihren Unterricht. Wahrscheinlich aus diesem Grund das Fehlen dieses Themas in ENEM (Silva et al., 2013).

Normalerweise teilen die Lehrpläne das Schuljahr der High School in vier Zweimonate auf. Jeder Zweimonatszeitraum entspricht einer Einheit mit jeweils 20 Stunden. In einem Jahr mit 80 Stunden wäre die Aufteilung dann gerecht. Einige Fächer brauchen jedoch länger als andere, um unterrichtet zu werden (Tabelle 2). Der Unterschied zwischen der geplanten Zeit und der Echtzeit für jede Einheit ist wahrscheinlich auf die fragmentierte Programmierung des Inhalts zurückzuführen. Auf diese Weise wird das Lernen entkettet, und der präsentierte, fragmentierte Inhalt weist verschiedene Schwierigkeitsfaktoren auf, für deren Fertigstellung weniger oder mehr Stunden erforderlich sind (Gaspar, 1997). Darüber hinaus wird die Unterrichtszeit in einem traditionellen Klassenzimmer auch für andere Aufgaben (Anruf, Notizen) und nicht für das Unterrichten der Disziplin selbst aufgewendet (Pires und Veit, 2006).

In der Referenzmatrix von Enem beziehen sich die meisten Untersuchungsobjekte auf die Mechanik. In derselben Matrix haben die Themen Welle, Optik, RC: 78530

Disponível em: https://www.nucleodoconhecimento.com.br/bildung-de/physik-am- 
Elektromagnetismus und Thermodynamik eine ähnliche Anzahl von Objekten. Die Präsenz dieser Themen in den Fragen ist jedoch nicht ähnlich. Sowohl in dieser Matrix als auch in den Fragen ist nur die Mechanik sehr präsent (Tabelle 3). Die Referenzmatrix umfasst im Thema Mechanik viele verschiedene Konzepte, die bei anderen Themen, beispielsweise der Welle, nicht vorkommen. Vielleicht verursacht diese von ENEM praktizierte Aufteilung, die sich von der Aufteilung der technischen Oberschule unterscheidet, den beobachteten prozentualen Unterschied (Brasil, 2015).

Einige Inhalte, die im technischen Kurs der Chemie am IFAP in der physikalischen Komponente vermittelt werden, haben beispielsweise eine Ladung von 80 Stunden (Elektrizität + Magnetismus) und entsprechen 7\% des in ENEM geladenen Inhalts. Andere, wie die Thermologie, werden in 40 Stunden in technischer Ausbildung unterrichtet und entsprechen $11 \%$ der nationalen Prüfung. Wieder andere machen als Welle $28 \%$ der ENEM-Bewertung aus und werden in 20 Stunden unterrichtet. Diese Ungleichheit tritt wahrscheinlich auf, weil die grundlegende Rolle von IFAP in dem oben genannten Kurs darin besteht, Techniker in Chemie auszubilden (Brasil, 2019). Dies bedeutet nicht, dass Schüler, die eine technische Sekundarschulbildung abgeschlossen haben, den ENEM nicht für den Hochschulzugang absolvieren.

Der Physikunterricht bereitet immer noch Schwierigkeiten hinsichtlich der Aufnahme von Wissen durch die Studierenden, was bedeutet, dass die meisten Studierenden in dieser Disziplin keine zufriedenstellenden Leistungen erbringen (Sbf, 2011; Barroso et al., 2018).

Der ENEM-Test sollte mit dem übereinstimmen, was der Schüler in der High School gelernt hat (normal oder technisch). Es konnte festgestellt werden, dass sich die Schwierigkeit von ENEM im Laufe der Jahre geändert hat (Abbildung 1). Der Versuch, die Schwierigkeit der nationalen Prüfungsfragen (2014 bis 2016) zu erhöhen, wurde in den folgenden zwei Jahren rückgängig gemacht. Da der Physikunterricht auf der Sekundarstufe immer noch eine geringe Leistung bei der

$\mathrm{RC}: 78530$

Disponível em: https://www.nucleodoconhecimento.com.br/bildung-de/physik-am- 
Wissensabsorption aufweist, hat dies möglicherweise die Entscheidung beeinflusst, den Schwierigkeitsgrad dieser Komponente in den Prüfungen 2017 und 2018 zu senken (Barroso et al., 2018).

\section{FAZIT}

Der Physikunterricht im technischen Kurs für Chemie am IFAP stellt keine Abteilung dar, die die in ENEM am häufigsten vorkommenden Fächer priorisiert.

ENEM kontextualisiert normalerweise seine Fragen. Dies könnte eine gängige Praxis in der Physik der High School sein, da dies zu Ihrem besseren Verständnis beitragen würde. Darüber hinaus ist es notwendig, den Inhalt während des Unterrichts oder als Komposition mit anderen Fächern nicht zu fragmentieren.

Der technische IFAP-Kurs wäre nicht der geeignete Studienort für diejenigen, die nur die High School beenden möchten. Der Inhalt geht über das Erforderliche hinaus, jedoch mit Ausschnitten, die auf den technischen Teil ausgerichtet sind, einschließlich Laborpraktiken und streng technischer Disziplinen.

Das Fehlen von Interdisziplinarität und Kontextualisierung macht es schwierig, den Inhalt aufzunehmen, was zu Studenten führt, die Schwierigkeiten haben, über Physik nachzudenken.

\section{VERWEISE}

BARROSO, M. F.; RUBINI, G.; SILVA, T. Dificuldades na aprendizagem de Física sob a ótica dos resultados do Enem. Rev. Bras. Ensino Fís., v. 40, n. 4, p. e4402, 2018.

BRASIL. Matriz de Referência Enem. Brasilia DF, 2015. Disponível em: < http://download.inep.gov.br/download/enem/matriz_referencia.pdf >. Acesso em: 25 mar 2020.

$\mathrm{RC}: 78530$

Disponível em: https://www.nucleodoconhecimento.com.br/bildung-de/physik-am- 
. Curso Técnico de Nível Médio em Química na Forma Integrada Regime Integral: Plano de Curso. Macapá AP, 2016. Disponível em: < https://portal.ifap.edu.br/index.php/publicacoes/item/1100-resolucao-n-20-2018consup >. Acesso em: 27 abril 2019.

. Expansão da Rede Federal. Rede Federal de Educação Profissional, Científica e Tecnológica. 2018. Disponível em: < http://redefederal.mec.gov.br/expansao-da-rede-federal >. Acesso em: 27 abril 2019. . Curso Técnico em Química - Integrado - Campus Macapá. Macapá AP, 2019. Disponível em: < http://www.ifap.edu.br/index.php/component/content/article?id=398 >. Acesso em: 27 abril 2019.

CASTRO, G. N. V. et al. Análise de Eficiência Acadêmica dos cursos subsequentes, nas modalidades à distância e presencial, ofertados pelo Instituto Federal do Amapá (2018). Research, Society and Development, v. 9, n. 8, p. e208985262, 2020. https://rsdjournal.org/index.php/rsd/article/view/5262

GASPAR, A. Cinqüenta anos de ensino de Física: Muitos equívocos, alguns acertos e a necessidade do resgate do papel do professor $X V$ Encontro de Físicos do norte e Nordeste Natal RN 1997.

MARQUES, J. D. C. et al. Nível Médio Técnico e Cursos de Graduação: comparativo de vagas e ingressantes no Instituto Federal do Amapá, Brasil (20172018). Research, Society and Development, v. 9, n. 8, p. e228985375, 2020 https://rsdjournal.org/index.php/rsd/article/view/5375

PACHECO, E. M. Os Institutos Federais: Uma Revolução na Educação Profissional e Tecnológica. Natal RN: IFRN, 2010. 28p.

$\mathrm{RC}: 78530$

Disponível em: https://www.nucleodoconhecimento.com.br/bildung-de/physik-am- 
PENHA, A. C. F. M. et al. Matrículas da Educação Especial na Educação Profissional Técnica de Nível Médio no Estado do Amapá (2015-2018). Research, Society and Development, v. 9, n. 7, p. e881974867, 2020. https://rsdjournal.org/index.php/rsd/article/view/4867

PIRES, M. A.; VEIT, E. A. Tecnologias de Informação e Comunicação para ampliar e motivar o aprendizado de Fısica no Ensino Medio. Revista Brasileira de Ensino de Fisica, v. 28, n. 2, p. $241-248,2006$.

RIBEIRO, J. L. P.; VERDEAUX, M. D. F. D. S. Atividades experimentais no ensino de óptica: uma revisão. Revista Brasileira de Ensino de Fısica, v. 34, n. 4, p. 1 10, 2012

SBF. Carta aberta ao Inep quanto à adoção do Exame Nacional do Ensino Médio como critério de ingresso nas Universidades. São Paulo SP, 2011. Disponível em: < http://www.sbfisica.org.br/v1/arquivos_diversos/noticias/maio2014/carta-aoINEP.pdf >. Acesso em: 05 fev 2021.

SILVA, J. R. N. D.; ARENGHI, L. E. B.; LINO, A. Porque inserir física moderna e contemporânea no ensino médio? Uma revisão das justificativas dos trabalhos acadêmicos. R. B. E. C. T., v. 6, n. 1, p. 1-15, 2013.

SILVEIRA, F. L.; BARBOSA, M. C. B.; SILVA, R. Exame Nacional do Ensino Médio (ENEM): Uma análise crítica Rev. Bras. Ensino Fís., v. 37, n. 1, p. 1101, 2015.

SILVEIRA, F. L.; STILCK, J.; BARBOSA, M. Comunicações: Manifesto sobre a qualidade das questões de Física na Prova de Ciências da natureza no Exame Nacional de Ensino Médio. Caderno Brasileiro de Ensino de Física, v. 31, n. 2, p. 473-479, 2014.

RC: 78530

Disponível em: https://www.nucleodoconhecimento.com.br/bildung-de/physik-am- 
TOTI, F. A.; PIERSON, A. H. C. Elementos para uma aproximação entre a física no ensino médio e o cotidiano de trabalho de estudantes trabalhadores. Investigações em Ensino de Ciência, v. 15, n. 3, p. 527-552, 2010

Gepostet: März, 2021

Genehmigt: März, 2021

RC: 78530

Disponível em: https://www.nucleodoconhecimento.com.br/bildung-de/physik-am$\underline{\text { enem }}$ 